Línea de Investigación,

\title{
1 \\ La relación entre conocimiento común y conocimiento científico en el contexto de la enseñanza, aprendizaje y cambio conceptual de las ciencias
}

Alfonso Claret Zambrano Ph. D.

\begin{abstract}
This paper analyses the research approach on the relationship between scientific scholar knowledge of the teacher and common previous knowledge of students in school within the context of teaching, learning and conceptual change in sciences. The paper shows two sections: the first is about conceptual historical development of the research question. In this sense the first question was How the students learn sciences and its transformation into the second, third, fourth and fifth question was justified on the light of the reading of the following works, mainly: Piaget, A usubel, Driver, Vygotsky, and Bachelard, Canguilhem, Kuhn, Lakatos, Popper and others authors. The second explains the research question taking into consideration the meaning of the teacher, the pupil and the scientific knowledge in the classroom. For this purpose it is necessary to design a conceptual structure in order to analyze the relations, the concepts and the research problems of the teaching, learning and assessment in sciences. The structure shows the relationship of the teacher and the pupilas knowledge relationship. This is the cause why scientific knowledge must be considered as the hard core of the science teaching. But scientific knowledge in this case is seen as a product of the its historical and epistemological development and the way as scientific knowledge changes in science is the basis forthinking about conceptual change of students in the classroom. The paper ends showing the aims of the researcher engaged in this approach.
\end{abstract}

\subsection{Antecedentes}

La educación en ciencias naturales es un campo en proceso de construcción. El origen de este campo data desde 1986 y su consolidación como campo de trabajo investigativo con problemas propios se hizo en 1988, con la realización de su segunda conferencia en Berkeley, School of Education, University of California.

Su punto de convergencia es la síntesis del trabajo de investigadores de Educación en Ciencias, investigadores en el campo de a cognición, la informática (especialmente aquellos que trabajan con el computador como elemento para diseñar como resuelven los alumnos problemas en ciencias, etc), los epistemólogos, los curriculistas y los educadores en ciencias.

La educación en ciencias, como área de investigación tiene acuerdo en ciertos problemas macros que orientan el trabajo investigativo y sirven de referencia al origen de esta nueva disciplina. Tales problemas, se ubican en cinco grandes líneas de investigación. La primera se orienta a solucionar los asuntos de la enseñanza de las disciplinas científicas y los estudios curriculares de las mismas. La investigación basada en las disciplinas y su desarrollo fue la actividad principal del campo educativo en 
Ciencias durante el período de reforma curricular de los años 50 y 60 (en Norteamérica y Europa) y aún continúa jugando un papel relevante en la actualidad.

Una segunda línea se refiere al trabajo de la ciencia cognitiva, cuyo campo, también en proceso de desarrollo, reúne elementos de la inteligencia artificial, sicología cognitiva, lingüística, filosofía y otras disciplinas. Su campo de investigación relacionado con educación en ciencias converge en los principios generales del aprendizaje, el conocimiento y el razonamiento. Desde los años 70 , este campo tiende a trabajar problemas comunes con educación en ciencias, tales como solución de problemas, conocimiento y aprendizaje de contenidos específicos de asignaturas. Actualmente hay un evidente intercambio de teoría y métodos empíricos en ambas disciplinas.

Una tercera línea está relacionada con el contexto social en el cual se desarrolla la enseñanza y aprendizaje de las ciencias naturales, un tópico cuya importancia es cada día mayor. Investigaciones acerca de la organización social de las escuelas, incluyendo actitudes socialmente determinadas hacia la escolarización y la participación en actividades de grupos, se benefician intensamente de los métodos y conceptos de las ciencias sociales y su ubicación con otras disciplinas de las ciencias naturales

Una cuarta línea analiza la tecnología educativa en las ciencias. Esta línea se refiere principalmente a la tecnología informativa producida por los computadores, videos, discos, telemática, gráficas, etc.

Finalmente existe una quinta línea cuyo interés de fondo es, investigar la relación entre el conocimiento común y el conocimiento escolar, como también entre razonamiento cotidiano y aprendizaje escolar. En este caso su tarea de fondo es resolver y construir modelos que expliquen e interpreten racionalmente el problema de la enseñanza y el aprendizaje de las ciencias. Esta línea de investigación que presentamos, se ubica en esta perspectiva.

\subsection{Justificación}

El objeto de esta línea de investigación es estudiar el mecanismo de la relación entre conocimiento común del alumno y conocimiento científico del maestro, en la enseñanza, aprendizaje y cambio conceptual de las ciencias a partir de la historia y epistemología de varios conceptos científicos que luego serán abordados desde una pedagogía constructivista basada en Vygotsky para su análisis. En este sentido se espera conocer como los alumnos de primaria, secundaria y universidad, de una localidad determinada, estructuran los contenidos y procesos de algunos de los conceptos de las ciencias naturales

Hay pocas referencias en el país de este tipo de investigación. Algunos autores han venido haciendo trabajos en esta área, pero limitándose al movimiento de concepciones alternativas. Otros como Segura (1991) han trabajado con la denominadas Actividades Totalidad abiertas (ATAS) buscando a través de las mismas la articulación entre el conocimiento previo del alumno y el conocimiento que se construye, así como el nexo entre el contenido del ATA y el desarrollo intelectual del alumno, además de la pertinencia de la actividad como medio de solucionar problemas de ciencias.

En Europa y Norteamérica existen va-ríos ejemplos, sin embargo, cierto número de ellos se ha limitado a identificar los pre-conceptos de los jóvenes sin avanzar más allá del simple diagnóstico. Algunos autores argumentan que el cambio de novato (o inexperto, 
esto es alumnos en proceso de formación educativa de pregrado) a experto envuelve la misma clase de cambio conceptual que sucede en la historia de las ciencias. Wiser (1983), por ejemplo, planteó dos propósitos como guía para su investigación; ambos se referían a las relaciones entre la teoría de la historia de las ciencias y la ontogénesis. El primero, trata del papel de la diferenciación como descripción de desarrollo en la historia de las ciencias y en ontogénesis (desarrollo del individuo). Wiser trató la diferenciación a través de un modelo histórico. En este caso ella tomó un modelo construido por científicos del siglo XVII, que no diferenciaba calor de temperatura, y lo usó para analizar si estudiantes inexpertos tenían un concepto similar. Su fin era ir más allá del diagnóstico y especificar a continuación la naturaleza del concepto de indiferenciación, sus características y relaciones a otros conceptos. El segundo era determinar si el contenido y estructura del modelo termal de los inexpertos era similar al modelo termal (recipientefuente) de los científicos aludidos. En ambos casos la respuesta fue positiva. Esto sugiere que el modelo de diferenciación de la historia de la ciencia es confirmado por el desarrollo de la ontogenesis. Wiser concluye una convergencia a nivel estructural pero no a nivel de contenido

Otros, a diferencia del autor anterior, han planteando que el contenido de la ciencia de los inexpertos es similar al contenido de la ciencia practicada en el pasado. Por ejemplo Disessa (1982) argumenta que la física del inexperto con relación a las leyes de Newton es aristotélica en el sentido de que los objetos se deben mover en la dirección que últimamente son empujados.

Estas tendencias de investigación inexperto-experto en solución de problemas y procesos de información brevemente se caracterizan por dos interpretaciones con valor educativo: La primera considera que el inexperto al solucionar problemas de ciencias muestra equivocaciones sistemáticas, a menudo interpretadas como falsas creencias. Lo crucial de esta asunción es considerar que el concepto del experto y el concepto del inexperto son lo mismo. El argumento de que el inexperto tiene una falsa creencia acerca del calor presupone que ambos experto e inexperto tienen el mismo concepto de calor (el del experto), incluyendo propiedades diferentes y relacionándolos con otros conceptos en forma distinta. Por ejemplo, Caramazza (1981) argumenta que estudiantes solucionando problemas simples acerca de la trayectoria de la calda de los cuerpos revelan una variedad de equivocaciones. Sin embargo las respuestas relacionan el contenido de la física del alumno inexperto a la física desarrollada de la época previa a Galileo. La persistencia histórica de estas ideas revelan que ellas son un producto de la vida cotidiana del niño.

La segunda considera que expertos e inexpertos difieren acerca de los procedimientos para solucionar problemas en ciencias y en proceso de información. La crucial asunción detrás de esta asunción es reconocer que el inexperto tiene su propia versión del problema, es decir, su propio concepto que no necesariamente es una versión degenerada del concepto del experto. Conocer su estructura, teoría, reglas y conceptos seria fundamental para la enseñanza y el aprendizaje de las ciencias.

Para Strauss(1988), la naturaleza e interpretación del debate inexperto-experto significa en el campo de la temática general del desarrollo dos posibilidades: la primera explorar el paralelo o convergencia entre el desarrollo de la anta-génesis, el desarrollo de la filogénesis y el desarrollo histórico de las ciencias. El segundo es acerca de cómo el desarrollo de la ontogénesis, filogéneis e historia de las ciencias pueden servir de heurística entre cada uno de ellos. Esta última dirección es la que orienta esta línea de investigación. Paralelo, significa secuencias de desarrollo longitudinal. Por ejemplo para 
un concepto histórico, $\mathrm{H}$, se necesitan dos puntos de una secuencia $\mathrm{H} 1$ y luego $\mathrm{H} 2$. Para un concepto ontogenético se necesitan dos puntos en una secuencia, $\mathrm{O} 1$ y luego $\mathrm{O} 2$ Por definición de similaridad entre $\mathrm{H} 1$ y $\mathrm{O} 1$ y luego entre $\mathrm{H} 2$ y $\mathrm{O} 2$ podemos argumentar un desarrollo paralelo entre el desarrollo histórico y el desarrollo ontogenético Convergencia atañe a similaridades entre $\mathrm{H} 1$ y $\mathrm{O} 1$ independientemente de su posterior desarrollo. Posner en E.U. hace una aproximación desde la Filosofía de las ciencias para crear un teoría de cambio conceptual basada en Lakatos y kuhn.

\subsection{El problema objeto de investigación: ¿cómo aprende el alumno en la escuela?}

El problema objeto de investigación en ésta línea tiene su propia evolución conceptual. Tal proceso implica conocer cual fue el primer problema objeto de reflexión y luego bajo que criterios teóricos se fue transformando en diversas alternativas hasta llegar al actual problema de investigación acerca de la relación entre el conocimiento común y el conocimiento científico. Para empezar, el primer interrogante del problema de investigación fue: ¿cómo aprende el alumno en la escuela? La forma como concebimos este problema se deriva de la historia epistemológica de las ciencias y no de la pedagogía. Al respecto Bachelard, filósofo francés, decía hace algunos años: Los profesores de ciencias se imaginan que el espíritu comienza como una lección, que siempre puede rehacerse una cultura perezosa repitiendo una clase, que puede hacerse comprender una demostración repitiéndola punto por punto. No han reflexionado sobre el hecho de que el adolescente llega al curso de Física con conocimientos empíricos ya constituidos; no se trata, pues, de adquirir una cultura experimental, sino de cambiar una cultura experimental, de derribar los obstáculos amontonados por la vida cotidiana." (Bachelard, 1975, p. 21).

El maestro no recibe un estudiante "vacio" para llenarlo con conocimiento empírico estructurado. Por el contrario él encuentra estudiantes con opiniones, con una ideología que ellos han adquirido y que los acompañará durante el desarrollo de sus vidas y a la cual ellos deben adaptarse con el fin de cumplir con las normas de una sociedad pero sin cuestionarse la razón de las mismas. Estas asunciones fueron objeto de reflexión y constituyen la razón de las siguientes preguntas: Si algunos puntos de vista suponen que los maestros enseñan a alguien que no sabe nada, porque Bachelard contradice la asunción del estudiante "vacío" ¿Consecuentemente como el estudiante aprende en la escuela? ¿Y cómo los maestros enseñan? Teniendo en cuenta las anteriores asunciones y preguntas la literatura fue revisada. Específicamente el trabajo de Piaget fue analizado con el objeto de ver que luz arrojaba en este asunto. Se obtuvo la siguiente conclusión.

Piaget (1972) estaba interesado en la Epistemología Genética y no en preguntas pedagógicas. El punto nodal del trabajo Piagetiano fue la construcción del pensamiento científico racional y la investigación psicológica en las operaciones mentales. Su pregunta central de investigación fue: ¿Cómo se pasa de un conocimiento correspondiente a cierta etapa del desarrollo a un conocimiento correspondiente a la siguiente etapa de dicho desarrollo? Esta pregunta no define a Piaget como maestro pero lo asocia con la Pedagogía. La respuesta de Piaget es completamente válida en el campo del desarrollo cognitivo del niño, pero no es posible un simple y puro traslado de la sicología a la Pedagogía sin previa elaboración teórica.

El punto central para esta pregunta fundamental acerca de cómo uno va de una etapa del desarrollo del conocimiento a la siguiente, es la proposición teórica de que la gente crea su propio conocimiento a través de sus propias acciones y la coordinación de esas acciones. Además, Piaget (1975) asume que el conocimiento es genético. En este sentido 
se lo considera como un proceso de desarrollo de tal manera que podemos estudiar su formación y progreso desde conceptos menores los cuales se vuelven parte de sistemas más y más elaborados. Por ejemplo saber que $2+2=4$ puede ser interpretado como una etapa genética porque no todos los sujetos poseen este conocimiento y puede ser estudiado con base en conceptos previas y por consiguiente ser objeto de desarrollo posterior.

Piaget (1964) diferencia entre desarrollo del conocimiento y aprendizaje. El primero es un proceso espontáneo atado al proceso total de embriogénesis incluyendo el cuerpo, el sistema nervioso y el desarrollo de las funciones mentales; el último es un proceso provocado por el sicólogo experimentador o el maestro. En ambos casos sin embargo hay un solo actor: la mente del niño construyendo por él mismo su propio conocimiento. En este sentido el aprendizaje es un caso particular del desarrollo del conocimiento; esto es, el desarrolla explica el aprendizaje. Como consecuencia de esta posición, el niña o niña solamente adquiere el conocimiento determinado por su propio desarrollo. Por consiguiente el aprendizaje es posible solamente cuando el niño tiene las competencias para lograrlo. Es decir, el mismo sólo es posible en el contexto del desarrollo del conocimiento propio del niño.

En otras palabras, el niño aprende cuando adquiere conocimiento actuando sobre los objetos y transformándolas: esto es, desplaza, une, combina, separa y reconstruye los mismos. Desde esta perspectiva la pregunta, ¿cómo el niña aprende? no tiene sentido y debe ser cambiada por una segunda pregunta de investigación: ¿cómo el alumno adquiere conocimiento en la escuela? Con esta nueva pregunta se releyó a Piaget y se encontró lo siguiente:

"Para entender el desarrollo del conocimiento es necesario comenzar con una idea central para mí. La idea de operación. Una operación es la esencia del conocimiento, es una operación interiorizada la cual modifica el objeto de conocimiento. Pero en adición, es una acción reversible: la cual puede tomar lugar en ambas direcciones. por ejemplo añadir o substraer, juntar o separar. Pero sobre todo una operación nunca es aislada. Esta siempre articulada a otras operaciones y como resultado es siempre parte de una estructura total.. .Y el problema central de desarrollo es entender la formación, elaboración, organización y funcionamiento de esas estructuras" (Píaget, 1964, p. 176).

De acuerdo con Piaget, esas estructuras tienen cuatro etapas de desarrolla: la primera es la sensoro-motor, etapa preverbal que dura aproximadamente los primeros veinticuatro meses de vida; la segunda es la etapa pre-operacional; la tercera etapa es la concreto operacional y la etapa final es la operacional formal o hipotética deductiva. Con base en lo anterior la segunda pregunta: ¿cómo el niño adquiere conocimiento en la escuela? Debe ser cambiada a una tercera pregunta de investigación: ¿cómo el alumna va de una etapa de conocimiento de cierta fase de desarrollo a la siguiente etapa de conocimiento correspondiente a la siguiente fase de desarrollo en la escuela? (El caso de esta línea de investigación).

Sabemos que el trabajo de Piaget estaba interesado en la epistemología genética o desarrollo cognitivo y muy poco en la educación o en la escuela. Por esta razón es legítimo diferenciar entre desarrollo cognitivo a conocimiento genético y conocimiento escalar el cual es el objeto del aprendizaje escalar. Pero el conocimiento escalar es altamente específico como puede verse en las asignaturas académicas tradicionales, tales cama, matemáticas, química, física, biología, etc. Sin embargo nuestro interés es conocer cómo el alumno construye dicho conocimiento en la escuela. Par otro lada nos referimos también al modo tradicional como los maestros escriben syllabus centrados en 
el contenido formal de la asignatura siguiendo preferiblemente una secuencia basada en la lógica de las ciencias que en las propias construcciones de los alumnas en esas materias. Cama resultada hay poca indicación de cómo el alumno progresivamente construye esas asignaturas. Por esa razón es necesario tener una idea completa acerca de cómo el alumna construye esos campos especificas del conocimiento si queremos resolver las diferentes relaciones entre conocimiento genético y conocimiento escolar.

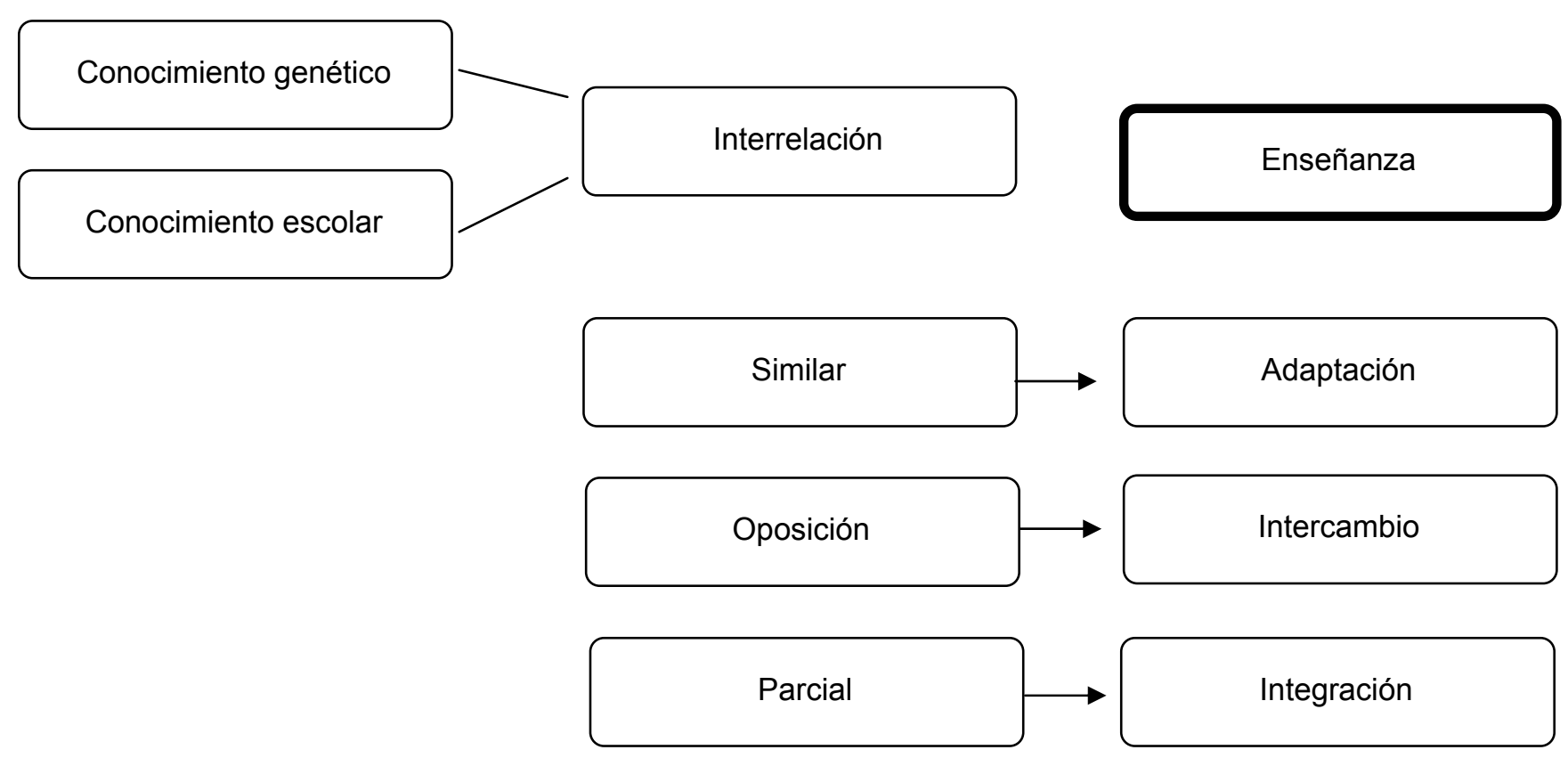

Figura 2. Las diferentes alternativas para enseñar como consecuencia de la interrelación entre conocimiento genético y conocimiento escolar.

El propósito teórico y práctico de analizar los procedimientos usados parlas alumnos para elaborar y construir el conocimiento escolar usando como base su conocimiento genético o desarrollo cognitivo, es un intento para delinear el papel del conocimiento genético en la apropiación del conocimiento escalar, por lo anterior la tercera pregunta de investigación se cambia por la cuarta pregunta de investigación fue elaborada así: ¿cómo el alumno obtiene conocimiento escolar o científico teniendo como base su conocimiento genético o conocimiento espontáneo previamente adquirido?

La figura 2 ilustra esta pregunta, si la interrelación entre el contenido del conocimiento genético y el contenido del conocimiento escolar es similar, el proceso de enseñanza es de adaptación entre ambos conocimientos. Si la interrelación es de oposición entonces el proceso de enseñanza es de intercambio del conocimiento equivocado por el conocimiento correcto. Si la interrelación es parcial entonces el proceso de enseñanza es de integración entre ambos conocimientos.

En términos de Piaget, la cuarta pregunta de investigación podría expresarse en el siguiente problema: Cómo es la relación entre el conocimiento genético o conocimiento espontánea adquirido por el alumno y el conocimiento escolar enseñado por el maestro en el contexto de la enseñanza, aprendizaje y cambio conceptual de las ciencias? Para 
avanzar en el análisis conceptual de este cuarto problema de investigación es necesario ampliar el significado del conocimiento espontáneo y el conocimiento escolar en el aula de clases

\subsubsection{El conocimiento espontáneo y el conocimiento escolar en el aula}

Inicialmente es necesaria aclarar que cuando nos referimos a frases como "conocimiento escolar" y "conocimiento espontáneo" estamos usando la diferencia establecida por Popper(1967) entre Mundo 2 y Mundo 3 Según Popper, Mundo 2 significa el mundo de nuestra experiencia consciente. Basado en esta apreciación concebimos que el alumno al llegar a la escuela trae sus propias ideas de ciencias (es decir, el conocimiento espontáneo) las cuales interrelacionan con el conocimiento escolar previo y sus concepciones sobre el mundo en general, integrándose en lo que hemos denominado, la estructura conceptual del alumno, la cual se materializa en el aula, como, el conocimiento común previo de los alumnos. En términos de Popper, Mundo 3 es el mundo del contenido lógico de libros, librerías, computadores. Con base en lo anterior concebimos que el Mundo 3 de Popper genera el conocimiento pública de las ciencias o el conocimiento científico, contenido en libros, revistas de investigación, textos de carácter científico y pedagógico, documentos, archivos, abstracts, y otros reconocidos por la comunidad científica nacional e internacional, el cual al ser apropiado pedagógicamente por el maestro (es decir, el conocimiento escolar) con el propósito de enseñar lo elabora de acuerdo a su formación curricular en ciencias, sus propias concepciones sobre ciencias, enseñanza y aprendizaje y su ejercicio teórico y práctico de las ciencias derivado de su pertenencia a instituciones educativas, integrándose en lo que hemos denominado, estructura conceptual del maestro, la cual se materializa en el aula, coma el conocimiento científico escolar. Parlas razones anteriores el problema a investigar en esta línea de investigación se cambia de la cuarta pregunta a una quinta pregunta: ¿cómo es la relación entre el conocimiento común previo del alumno y el conocimiento científico escolar del maestro en el contexto de la enseñanza, aprendizaje y cambio conceptual de las ciencias?

Pero la pregunta anterior exige explicar el significado y las relaciones entre los elementos involucrados en la misma, para determinar la estructura conceptual que enmarca el objeto de investigación en el aula y los problemas que se originan a partir de esta consideración. (Ver Figura 4).

\subsection{La estructura conceptual y los problemas fundamentales a investigar en la relación entre conocimiento común previo y el conocimiento científico escolar en el aula de clases}

La relación pedagógica fundamental entre el maestro, sujeto de la enseñanza y el alumna objeto del aprendizaje, es la problemática del planteamiento, la re-producción, apropiación y aplicación del conocimiento científico. Esta racionalidad es clave para entender la epistemología de la enseñanza, aprendizaje y evaluación de las ciencias.

Por esa razón el problema de la enseñanza-aprendizaje-evaluación sala se puede entender tomando coma referencia conceptual el conocimiento científico en la escuela.

Tal conocimiento esta dado par los contenidas científicos de la práctica científica; la estructura conceptual del maestra, que comprende los contenidos científicos apropiados pedagógicamente por el maestra can el propósito de enseñar; la estructura conceptual del 
alumno, que comprende las contenidos científicos previas apropiadas espontáneamente por el alumna; la secuencia de conocimientos científicos para la enseñanza aprendizaje; el esquema del proceso enseñanza-aprendizaje y evaluación. La anterior implica que para la enseñanza-aprendizaje-evaluación de los conceptos es necesario investigar varios problemas.

1.4.1 Conocer la estructura conceptual del maestro sobre los conceptos científicos (o conocimiento científico) de la práctica científica. En este sentido el maestro(a) lo reconocemos como el sujeto de la enseñanza, y lo asumimos con base en su estructura conceptual, tal cama se definió en la sección 1.3 .1 y la cual pedagogicamente se materializa en el conocimiento científico escolarizado para asumir la enseñanza, aprendizaje y evaluación de las ciencias en el aula de clases. Es decir, reconocemos que hay una epistemología coherente entre la que el maestra piensa que es su estructura conceptual sobre las ciencias en el orden científico y pedagógica y la forma como actúa en el aula. Así, si epistemológicamente concibe la existencia de la ciencia cama resultado o abstracción o esencia de las distintas prácticas científicas y por consiguiente válidas para cada una de ellas; entonces concibe y práctica la enseñanza en forma similar para la física, la historia o las matemáticas. Esto significa, que desconoce, que existen las ciencias y no la ciencia en singular, las cuales son diferenciadas, tienen un desarrolla desigual, y por consiguiente tienen una pedagogía particular. 
Estructura conceptual en la educación en ciencias

problema

Plantear, reproducir, apropiar y aplicar el conocimiento científico

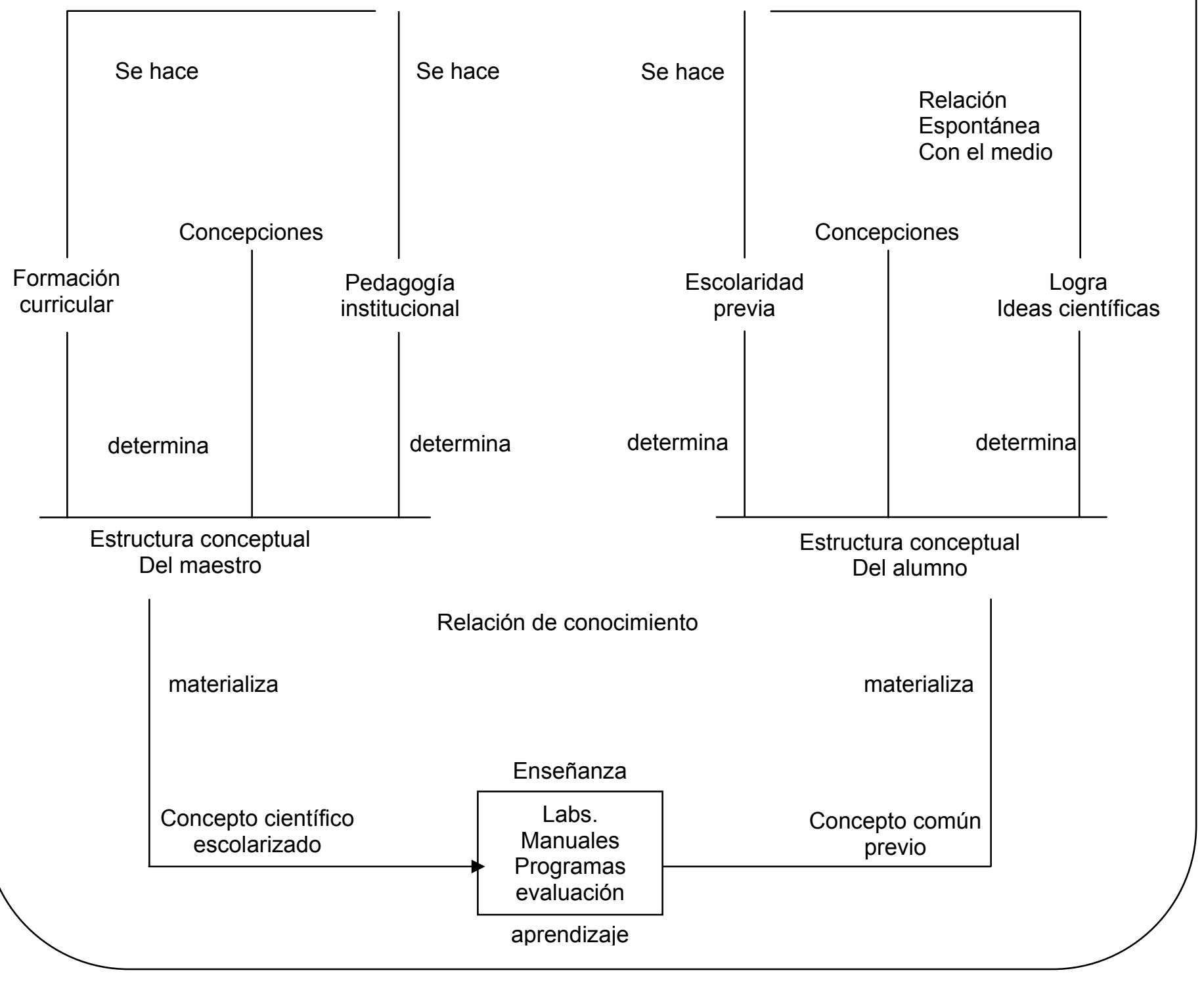

Figura 4. Estructura conceptual de la enseñanza, aprendizaje y evaluación de las ciencias

Por otro lado si se considera que las ciencias son objetivas, neutras y precisas en sus resultados lleva a darle a la enseñanza un carácter dogmático, acabado y cerrado y a concebir la misma únicamente como un asunto de resultados, hacienda a un lado el proceso que los produjo.

Usualmente para algunos científicos y maestros es difícil comprender el valar pedagógico de la enseñanza de un concepto de las ciencias en el marco de todas las relaciones sociales, económicas, políticas, históricas, ideológicas que coexisten en el origen del mismo, porque conciben que únicamente los resultados de las ciencias tienen validez absoluta para la misma y que las otras relaciones solo son obstáculos para su enseñanza y apropiación. En tal sentido plantean la neutralidad de las ciencias, cuando 
precisamente un concepto científica se forma se rectifica y progrese en el contexto de un haz de relaciones semejantes.

Si por el contrario epistemologicamente se conciben las ciencias como un conjunto de relaciones históricamente determinadas de conceptos sistematizados en un campo conceptual donde teórica y metológicamente abordan en aproximaciones sucesivas, cada vez mas objetivas la explicación racional de un sector de la realidad, se comprende porque la pedagogía de una ciencia en particular, según esta concepción tiene que empezar por conocer su propia práctica científica, y no la ciencia en general. Nos obliga a remitirnos a la historia y explicación de los conceptos de cada ciencia y además nos lleva a expresar los mismos como razón y como experiencia, cama teoría y coma hecho. Por tal razón un concepto, debe participar de la conceptualización teórica y su correspondiente aplicación práctica en una continua relación.

Con base en lo anterior es problema fundamental para la enseñanza de las ciencias conocer acerca de las concepciones del maestra por el carácter determinante que juegan en su teoría y práctica educativa en la escuela y en la aplicación de su conocimiento escolarizado en el aula.

1.4.2 Identificar la correspondiente estructura conceptual del alumno sobre los conceptos científicos (conocimiento científico) de la práctica científica. El alumno (a) lo reconocemos coma el sujeta del aprendizaje y lo asumimos con base en su estructura conceptual, tal como la definimos en la sección 1.3.1 y la cual se materializa en el conocimiento común previo, que necesariamente van a interferir en forma positiva o negativa con la enseñanza en la escuela. La apreciación de este hecha no es nueva ${ }^{1}$. Pero un nuevo desarrollo de esta apreciación es el esfuerza sistemática de aplicar el conocimiento común previo de los alumnos a la enseñanza, aprendizaje y evaluación de las ciencias naturales en la escuela

Varios problemas se identifican en este contexto. A. Un primer problema tiene relación con la diversidad de la terminología asignada a las ideas de los alumnos sobre el mundo socio natural asumiendo que cualquiera sean los términos que se usen tienen igual significado y por consiguiente son equivalentes. Pero en el fondo lo que se esta haciendo es eludir el marco teórica y los conceptos relevantes que se deriven de las principios conceptuales que se están aplicando. Términos como concepciones erróneas (misconceptions en Helm, 1980), preconcepciones (preconceptions en Ausubel, 1968), ciencias de los niños (children's science en Gilbert, Osborne y Fesham.1982) marcos alternativos (alternative frameworks en Driver, 1981), razonamiento espontáneo (spantaneous reasoning enVienot, 1979),. representaciones (representations en Giordan, 1978), preteorias(Segura, 1981), etc. muestran la dificultad de pensar un nombre común para todos los hallazgos investigativos en este asunto expresando en el trasfondo compromisos teóricos y prácticos que indican la posición filosófica que subyace en el uso de ciertos términos y no otros.

B. Un segundo problema, se refiere a reducir la investigación de las conceptos previos de las alumnos al simple diagnóstico, limitando sus resultados, únicamente, al proceso de su identificación, estudio y caracterización. Tales estudios fueran frecuentes durante la década del setenta. Driver (1978) por ejemplo asume das interpretaciones para las conceptos previos de los alumnos. En la primera, los compara con las ideas aceptadas de las ciencias y los califica por su congruencia con las mismas. A este grupo también

\footnotetext{
${ }^{1}$ Vico en 1710 consideraba que el criterio y regla de lo verdadero es haberlo hecho. 
pertenecen los términos: preconcepciones (Ausubel,1968), teorías noveles (McCloskey,1983), concepciones erradas, etc. definidas por algunos investigadores de la enseñanza de las ciencias para mostrar las diferencias entre los conceptos propios de los alumnos, asumidas como un conocimiento equivocado y los conceptos científicas asumidos como un conocimiento correcto (Helm, 1980). De acuerda con esta interpretación, los conceptos previas de los alumnos son sorprendentemente te-naces y resistentes a la extinción. En contraste con la primera interpretación, la segunda se refiere fundamentalmente a las comprensiones propias de los alumnos en las cuales sus propias conceptualizaciones son exploradas y analizadas en sus propios términos sin ninguna evaluación contra un sistema externo definido. En esta segunda interpretación se aceptan los conceptos previos de los niños tal cama ellos son, sin hacer ningún juicio comparativo con las ideas de las ciencias. A este grupo pertenecen términos tales como: marcos alternativos (Driver, 1981), ciencia de los niños (Gilbert, 1982), etc.

Una interpretación alternativa a las dos anteriores considera que lo importante no es comparar los conceptos previos de los alumnos ni reconocer su singularidad, sino usarlos mismas en el pro-cesa enseñanza aprendizaje de las ciencias. Esta alternativa constituye una fuente rica de investigaciones apoyadas par los planteamientos de Ausubel, Driver y Vygotsky (Zambrano, 1996).

C. Un tercer problema es planteado por Ogbarn (1975) quien crítica el problema anterior y propone como alternativa buscar teorías sobre el contenida de los conceptos previas de los alumnos.

El mismo construyó una teoría sobre dinámica para explicar algunas concepciones que tenían los alumnos en algunas situaciones en las cuales concebían la fuerza del movimiento actuando en un objeto móvil, pero en otras ocasiones pensaban las fuerzas como la acción de un objeto sobre otro. Su teoría trata de explicar por qué surgen estas concepciones particulares y no otras. Su primera etapa fue proponer una versión de la teoría del movimiento de donde se derivan esas concepciones. Esa teoría incluía términos como: apoyo, caída, lugar y camino y reglas de procedimiento. Por ejemplo los cuerpos pesados y a mayor altura caen más rápido. Construirlas teorías de los distintos dominios de las ciencias trae enormes ventajas para la investigación del aprendizaje en esta área. Viennot (1979) siguiendo esta dirección construye un modelo de cómo el alumno razona problemas de mecánica newtoniana.

D. Un último problema analiza la efectividad del cambio conceptual en la enseñanza y aprendizaje de las ciencias (Hewson, 1985, Strauss, 1987). Aquí es menester aclarar las relaciones y diferencia entre aprendizaje y cambio conceptual. El aprendizaje se asume como el resultado de articular el proceso teórico-práctico del conocimiento común del alumno y el conocimiento científico del maestro. Dado que el propósito final del aprendizaje es cambiar los conceptos propios de los alumnos parlas conceptos científicos de las ciencias, es necesario tomar como punta de referencia de este proceso el cambio conceptual en historia y epistemología de las ciencias. En este sentido asumimos que el estudio del cambio conceptual en la historia de las ciencias puede iluminar la comprensión del maestro sobre el aprendizaje de los conceptos de las ciencias en la escuela. Esta suposición considera varios puntos. Primero, ambas actividades son racionales. Tanto los científicos como las alumnos están envueltos en la misma clase de actividad: solucionar problemas usando ciertos conceptos previos para transformar su conocimiento actual. Segundo, ambos procesos tienen una meta idéntica: adquirir conocimiento objetivo. En ambos casos la objetividad del conocimiento requiere una ruptura con el conocimiento común. Desde este punta de vista el progreso del conocimiento acerca de un objeto, 
concepto o teoría consiste en argumentar contra su concepto previo destruyendo su conocimiento pasado" o superando los obstáculos que se oponen a ello. Esto sugiere que el alumno en el aula es cama el científico en las fronteras de las ciencias. Aquel cambiando su propio pensamiento y éste avanzando en el conocimiento científico pera ambas tratando de entender y explicar la realidad en sus propios términos con el fin de adquirir conocimiento. Teniendo en cuenta lo anterior, en sentido estricta, cambio conceptual aplica únicamente a las ciencias y en este caso lo utilizamos como una heurística para analizar el aprendizaje en la escuela. Pera dada la similitud entre los dos procesos, en sentido amplio, podemos hablar de cambio conceptual en la escuela pero haciendo hincapié en la naturaleza diferente de este última proceso, con relación al de las ciencias, de allí la particularidad del aprendizaje para estos casas. Varias concepciones asumen el estudio, análisis e investigación del cambia conceptual basándose en algunas de las diferentes posiciones canstructivistas, a en algunas de las posiciones asociacionistas. Otros investigadores teniendo como referencia las concepciones alternativas de Driver (1978) colocan en duda la efectividad del cambio conceptual.

De lo dicho anteriormente queda claro que los alumnos "piensan" y piensan diferente de los adultos. Obviamente hay diferentes problemas al respecto. Es tarea fundamental de la enseñanza y aprendizaje de las ciencias partir de aquello que el alumno sabe y tiene experiencia.

1.4.3 Esclarecer el significado y las relaciones de los conceptos científicos y su integración con las estructuras globales de la práctica científica. El concepto científica es el objeto del proceso enseñanza-aprendizaje y evaluación. Conocer su naturaleza, sus características y su contenido lógico organizado en materiales impresos de carácter público son fundamentales por el papel que el mismo juega en las concepciones del maestra y el uso que del mismo se hace en el aprendizaje del alumno(a). Con el término nos estamos refiriendo a los distintos conceptos de las prácticas científicas. En este caso, si les definimos asumiéndolas ubicadas en el contexto de las prácticas sociales de una sociedad determinada, como el proceso de transformación que se realiza a partir de hechos, ideas, representaciones, nociones, conceptos, teorías por medio del trabajo del hombre sobre la naturaleza y el uso de los métodos determinados y cuya producto más esclarecido es el conocimiento científico, entenderemos porque al hablar de este último nos estamos refiriendo al conocimiento biológico, físico o químico. Ya se trate de cualquiera de esta disciplinas estos conocimientos científicos son el resultado de un proceso de transformación a través de medios determinados que hacen que los mismos tengan una especificidad, una diferencia y un desarrollo desigual como práctica científica.

En este sentido las ciencias con relación a lo anterior tienen su propio objeto, su propia teoría, su propio método y su propios protocolos experimentales particulares y con relación a su desarrollo desigual su propia historia y por consiguiente su propia pedagogía que se inscribe en una relación necesaria y específica can cada una de dichas prácticas científicas, las cuales la determinan en última instancia. De allí expresamos, el porque, no existe una pedagogía para la ciencia, sino una pedagogía de la química o una pedagogía de la biología. Esta proposición epistemológica nos permite recuperar la construcción de una pedagogía desde el interior de cada práctica científica, uniendo lo que la teoría pedagógica positivista tanta ha separado la ciencia y la pedagogía, o el método de investigación y el método de exposición. Esta suposición epistemológica es la que nos permite entender porque hay una relación indivisible, necesaria y suficiente entre las ciencias respectivas y su enseñanza correspondiente, parlo tanto la indivisibilidad de la investigación y la docencia de cada una de estas disciplinas constituyen su estructura epistemológica normal. 


\subsubsection{Acerca del conocimiento científico y su pedagogía}

La concepción del conocimiento científico en un contexto socialmente determinado implica reconocer que la enseñanza de los resultados de las prácticas científicas no es una enseñanza científica, sino que se hace explicito el contexto epistemológico o explicativo o teórico e histórico que ha producido dicho resultado.

El mejor ejemplo de lo que es esta pedagogía lo ofrece Canguilhem, al analizar el experimento, coma la alternativa pedagógica en la enseñanza de las ciencias, cuando se toma coma un simple resultado fuera del contexto de proceso del conocimiento epistemológico e histórico en el cual tiene sentida." Nos explica en una lección acerca de la contracción muscular que el profesor está muy feliz por haber establecido un hecha cuando montó la experiencia clásica que consiste en aislar un músculo en un tarro lleno de agua y en mostrar que bajo el efecto de una excitación eléctrica el músculo se contrae sin variación del nivel liquido". De este" hecho" concluirá: la contracción es una modificación de la forma del músculo sin variación del volumen. Canguilhem, comenta: Es un hecha epistemológico que un hecha experimental enseñado de esta manera no tiene sentida biológico. Para dar sentido a este hecha hay que reman-terse al primero que tuvo la idea, de una experiencia de este tipa, es decir, a Swammerdan, (1637-1680 ) contra las teorías de origen galénica y estoico entonces dominantes, se trataba de demostrar que el músculo de rana aislado continuaba respondiendo a la excitación del nervio mucho tiempo después de la interrupción de la relación con la medula espinal, y además la contracción se hacia sin cambio de volumen y sin aumento de sustancias todo esto se oponía a contracción por flujo de los espíritus animales en el músculo. Alejado de este debate, fijado en una pedagogía sin historia este "hecho" pierde su sentido real, en verdad histórico para ubicarse en las melancólicas disertaciones sobre "método experimental con las que se complace cierta epistemología dogmática" ${ }^{2}$

\subsubsection{El proceso discontinuo del conocimiento científico}

Paralelamente a lo anterior, no se puede concebir la práctica científica cama un proceso de transformación acumulativa, en el cual los resultados pertinentes son simplemente una continuidad que va del conocimiento común al conocimiento científica y por ende de la simple a lo compleja, de lo concreto a lo abstracto, de lo conocido a lo desconocida.

Por consiguiente develar la continuidad del conocimiento científico es asumir el mismo coma un proceso discontinuo, esto es como una ruptura entre el conocimiento común y el conocimiento científico. Par tal razón hay que plantear el problema del conocimiento científico en términos de obstáculos. Tal como Bachelard la asume: "Se conoce en contra de un conocimiento anterior, destruyendo conocimientos mal adquiridos o superando aquella, que en el espíritu mismo obstaculiza a la espiritualización". Es por esta tesis Bacherladiana que pensamos que la enseñanza científica sala es pasible a partir de superar las obstáculos epistemológicos y pedagógicas que se oponen a su realización.

\subsubsection{La organización lógica del conocimiento}

La tesis fundamental en torna a este punto consiste en reconocer la especificidad del mundo tres de Popper, esto es, el mundo de las contenidos lógicos en libros, librerías, computadores. En este sentido la presentación pública del conocimiento tiene una lógica

\footnotetext{
${ }^{2}$ Para una crítica a la Epistemología-Dominique Lecourt, Editorial Siglo XX Colección Mínima, pp. 70-71
} 
particular que es pertinente dilucidar para una mejor enseñanza. Por ejemplo, el conocimiento científico en su relación con la práctica científica se presenta en estructuras. Esto es, su contenido, los conceptos de jas ciencias no se presentan aislados; se presentan organizados en sistemas de conceptos o conocimientos relacionados. La estructura incluye el planteamiento de sus problemas, medición e interpretación de los mismos en términos de sus teorías fundamentales, la organización de sus conceptos, su orden, jerarquía, relaciones, hechos y métodos. Saber de las redes conceptuales entre los conceptos de las ciencias se convierte en un problema fundamental para la enseñanza de las ciencias.

1.4.4 Resolver cómo es la interrelación entre los conceptos previos comunes de los alumnos y los conceptos científicos escolarizados" del maestra. Para la enseñanzaaprendizaje de las ciencias es tarea fundamental resolver este problema. Epistemológicamente este asunto significa: cómo el alumna construye su propio conocimiento de la disciplina a partir de sus concepciones y las del maestro. Pedagógicamente significa las actividades y estrategias que el maestro crea para posibilitar el encuentro racional entre las ideas de los alumnos y sus ideas en el aula de clases. Según la teoría y la práctica que se asuma sobre las relaciones entre la enseñanza y el aprendizaje se originan diferentes interpretaciones para su materialización en el aula de clases.

\subsection{Propósitos}

Esta propuesta de línea de investigación permite saber qué es lo que el estudiante ha entendido de dichos conceptos antes y después de su enseñanza respectiva. Además nos permitirá establecer las condiciones pedagógicas en las cuales es pasible enseñar y aprender dichos conceptos basados en una concepción histórica y una concepción pedagógica constructivista de las ciencias.

En particular como producto conceptual de esta línea se espera: conocer el mecanismo como el conocimiento común de los alumnos interactúa con el conocimiento científico escolar de los maestros, de tal forma que el proceso enseñanza, aprendizaje y cambio conceptual sea más efectivo a los propósitos pedagógicos de la escuela y del país, Saber acerca de cuál es el proceso de formación de los conceptos a largo de la vida escolar de las alumnos, Analizar el proceso de enseñanza a partir de conocer el proceso del aprendizaje de los alumnos, Elaborar alternativas pedagógicas de orden curricular que tengan en cuentan el uso de los conceptos previos de los alumnos de su vida cotidiana cama de su vida escolar.

\section{Bibliografía}

AUSUBEL, D. P. (1968). Educational Psychology: A cognitive view, Halt Rinehart and Winston Inc., London.

BACHELARD, G. (1975). La Formación del Espíritu Científico, Editorial Siglo Veintiuno, Buenos Aires.

BRUNER, J. S. (1968). The Process Of Education, Harvard University Press, Mass: Cambridge.

CARAMAZZA, A. and OTHERS. (1981). Náive belíefs in 'sophisticated' subjects: Misconceptíons about trajectories of objects, Cognition: 117- 123. 
DISSESA, A. (1982). Unlearning Aristotelian Physics: A Study Of Knowledge. Based Learning, Cognitive Sciences, 6, 37-75.

DRIVER, R (1981) Pupils alternative frameworks in Science. Eur. J. Sci. Educ 3. 93-101

DRIVER R AND RUSSELL, (1981). An investigation of the ideas of heat, temperature and change of state of children aged between 8 and 14 years, Unpublished manuscript, University of Leeds, cited in Childrens ideas of science. edited by Driver, R. and Guesne E and Tibesrghen A.(1985), Open university. London

DRIVER, R.. and EASLEY, J. (1978). Pupils and paradigms: a review of literature related to concept development in adolescent science students, Studies in Science Education, $5,61-84$.

GILBERT, J. K OSBORNE, J. R. and FESHAM, P. J. . 3. (1982). Children's science and its consequences for teaching Science Education, 66(4), 623-633.

HELM, H (1980). Misconceptions in physics amongst South A frican pupils stufying physical science, South African Journal of Science, 74, 285-290.

HEWSON , M. G. A. B. (1985). The role of intellectual environment in the origin of conceptions: An exploratory study. In Cognitive structure and Conceptual change, edited by West, L. H. T. and Pines, A. L., Academic Press, London.

HEWSON, M. G. A B (1982). Students existing knowledge as a factor influencing the acquisition of scientific knowledge, Ph.D. Universrty of the Witwayersrand, South Africa.

HEWSON, P. W (1981). A conceptual change approach to learning science, Eur. Sci. Educ., 3(4), 383-396.

INHELDER,,B.,SINCLAIR H. and BOVET, M. (1974). Learning andDevelopmentin Cognition, MA Harvard University Press, Cambridge.

LECOURT, D (1975). Marxism ond Epistemology: Bachelard Canguilhem, Foucault, translated from the French by Ben Brewster N.L.B.

OGBORN , J. (1985). Eur. J. Sci. Educ., Vol. 7, No 2,141- 150.

PIAGET, J. (1964). Development and Learning, Journal of Research in Science Teaching, 2, 176-186.

PIAGET, J. (1971). Biology and knowledge, The University of Chicago Press, Chicago.

PIAGET, J. (1972). Psychology and Epistemology, Pínguin University books.

PIAGET, J. (1975). Introducción a la Epistemología Genética: El pensamiento matemático, Editorial Raídos, Buenos Aires.

POPPER, K (1967). Ponencia presentada el 25 de agosto de 1967 al tercer Congreso Internacional de Lógica, Metodología y Filosofía de la Ciencia en Conocimiento Objetivo Editorial Tecnos SA. 1988 Madrid. 
SEGURA, D. (1981). El aprendizaje de la ciencia a nivel básico: ¿continuidad o discontinuidad? En Naturaleza Educación y Ciencias $\mathrm{N}^{0} 0$.

STRAUSS S. and STAVY, R. (1983). Developmental Psychology and Curriculum Development: The case of heat and temperature. Paper presented at the international seminar (Misconceptions in Sciences and Mathematics) held at Cornell University.

STRAUSS, S. and STAVY, R. (1982). Ushaped behavioural growth: Implications for theories of development, In W. W. Hartup (Ed.), Review of child development research, 6, 547-599. University of Chicago Press, Chicago.

STRAUSS, S. (1987). Educat,onal developmen tal Psychology and School Learning. En LIBEN, S. L. (1987) Development and Learning: Con flict or Con gruence, Lawrence Erlbaum Associates, Publishers New Jersey.

VIENNOT, L. (1979). Spontaneous Reasoning in Elementary Dynamics, Eur. J. Sci, Educ., Vol 1, No 2, 205-221.

VYGOTSKY, L. S. (1962). Thought and language, MA: MIT Press, Cambridge. 209.

WISER, M. and CAREY, S. (1983). When heatandtemperature were one. En Mental Models editado por Dedre Gentner and Albert, L. Stevens lawrence Erbaum Associates, Publishers, London, 267-297.

WISER, M. (1983). The differentiation of heat and temperature: History of science and novlce-expefl shift. Publicado en Strauss, S. (1988), Ontogeny, Phylogeny, and Historical De velopment, Human Development, Volume 2, Tel Aviv University, Ablex Publishing Corporatrion Norwood, New Jersey.

ZAMBRANO, A. C. (1996). El Constructivismo según Ausubel, Driver y Vygotsky, Actualidad Educativa, Año III, No. 12. 